very reluctant to discuss eye donation as they perceive it as something that will distress patients and family members.

Aim This presentation shares findings regarding the views of patients and carers, including: their feelings and thoughts about the option of eye donation being raised with them, who they think should raise this issue, when this option should be discussed and who should be included in the discussion. Findings are drawn from the NIHR funded national study: Eye Donation from Palliative and Hospice care contexts: investigating Potential, Practice, Preference and Perceptions taking place in three palliative care and three hospice care settings across England (EDiPPPP) and the global literature.

Conclusion In view of data from the Royal National Institute of Blind People (RNIB, 2016) reporting that over two million people in the UK are living with sight loss and their prediction that this figure will double to nearly four million by 2050 it is imperative that anyone who could be, and would want to donate the gift of sight, is offered the opportunity to do so, especially as they approach their end of life.

\section{0-20 LIVING WELL WITH AN ADVANCED LUNG CONDITION}

Sally Boa, Lee Deane, Robyn Smith, Jackie Higgins, Sarah Miller, Holly McGuigan, Alison Moodie. Strathcarron Hospice, Denny, UK

\subsection{6/spcare-2021-Hospice.19}

Background Whilst our established support for people living with breathlessness was long-standing, it was not multidisciplinary or fully evidence-based. We wanted to address this for the benefit of patients and the wider health system. The Cambridge Breathlessness Intervention Service (CBIS) model is an evidence-based approach to support people living with advanced lung conditions to manage their breathlessness (Higginson, Bausewein, Reilly, et al., 2014). Consultation with service users suggested that patients wanted support to: manage breathlessness, improve mental wellbeing, mobility and symptom management as well as help with planning ahead and reducing isolation.

Aims To implement and evaluate a breathlessness intervention using a multidisciplinary skill mix and trained community volunteers.

Methods Using the CBIS model as a framework, we reshaped, redeployed and trained our team to deliver this intervention at home. We supplemented this with a layer of social support provided by experienced compassionate neighbour volunteers. We evaluated the impact of the intervention through: before and after breathlessness self-rating scales, interviews with patients and case studies documenting the use of different parts of the service (e.g. physiotherapist, nurse, occupational therapist, rehabilitation assistant, complementary therapy).

Results This project has been delivered during the COVID-19 pandemic, so there were practical issues around seeing patients face-to-face, and we were unable to support as many as expected. To date, 28 patients have been referred to the service. Of these, 18 received input. We will report on changes in self-rating scores and feedback from interviews with patients and families. To date, only a few participants opted to have a compassionate neighbour.

Conclusions Initial findings suggest that although delivery of this project was hampered by the pandemic, patients valued the service and benefitted from practical input from the MDT.
Limitations on being able to provide face-to-face support may have influenced uptake of compassionate neighbours and reduced the number of patients seen.

\section{Parallel Session 6.1 - Supporting children and young people: addressing choices and challenges (Friday 5 November, 13:00 - 14:00)}

\section{0-21 'EXHAUSTED, EMOTIONAL, AT BREAKING POINT': THE MENTAL WELLBEING OF YOUNG ADULTS WITH SHORTENED LIVES}

${ }^{1}$ Sarah Earle, ${ }^{1}$ Maddie Blackburn, ${ }^{2}$ Liz Chambers, ${ }^{3}$ Julia Downing, ${ }^{4,5}$ Kate Flemming ${ }^{5}$ Jamie Hale, ${ }^{1}$ Hannah Marston, 'Lindsay O'Dell, ${ }^{6}$ Sarah Earle, ${ }^{7}$ Lucy Watts, ${ }^{8}$ Sally Whitney. ${ }^{1}$ The Open University, Milton Keynes, UK; ${ }^{2}$ Together for Short Lives, Bristol, UK; ${ }^{3}$ ICPCN, Bristol, UK: ${ }^{4}$ Hospice UK, London, UK: ${ }^{5}$ Department of Health Sciences, University of York, UK; ${ }^{6}$ Independent Researcher, London, UK; ${ }^{7}$ Independent Researcher, Essex, UK; IIndependent Researcher, Brighton, UK

\subsection{6/spcare-2021-Hospice.20}

Background Young adults with life-limiting or life-threatening conditions (LLTCs) are ordinarily extremely challenged in terms of health and wellbeing and especially vulnerable to social isolation and exclusion (Johnson \& Hodgson, 2018; Knighting K, Bray L, Downing J, et al., 2018). Society's response to COVID-19 poses an additional threat to this group who are at risk of being further left behind, creating long-lasting consequences for their mental wellbeing.

Aims To examine the unintended consequences of (COVID19) epidemic-control decisions on young adults with LLTCs to (a) understand the needs of this group (b) develop guidance and support and, (c) determine how consequences could be mitigated.

Methods This inclusive research project, underpinned by an interpretive qualitative framework (Denzin, 2003) draws on the principles of co-design (Nind, 2014). Research participants were recruited using purposive and theoretical sampling using a variety of methods including advertising for participants, existing networks and snowballing. During July-September 2020 , in-depth interviews were conducted via video-conference with 28 young adults aged 18-40. Data were analysed iteratively using constructivist Grounded Theory Method (cGTM) (Charmaz, 2006) and using the qualitative software analysis package QSR NVivo.

Results Participants experienced a decline in mental wellbeing with those with pre-existing mental health issues showing the most significant decline; in these cases, participants spoke about suicidal ideation, depression and anxiety and an increased need for medication and therapy. Many participants described a 'rollercoaster' of emotions and said that they had felt 'petrified' 'overwhelmed, 'worried' and 'forgotten'. Key themes included (a) the constant management of risk to avoid (physical) harm from COVID-19 while protecting mental health (b) the desire to 'keep busy' as a means of 'thinking less' in order stay mentally well (c) a re-evaluation of what matters in life.

Conclusion Main learning points: (1) acknowledgement that although people with LLTCs are typically isolated and excluded that the pandemic has magnified and exacerbated 
this; (2) the need for regular communication; (3) enhanced mental health support.

\section{0-22 THE IMPACT OF A NEW CHILDREN'S PALLIATIVE CARE SERVICE ON PLACE OF DEATH: GIVING FAMILIES CHOICES}

1,2Joanna Elverson, ${ }^{1}$ Helen Aspey, ${ }^{2}$ Owen Lever, ${ }^{1}$ Ellie Bond ${ }^{1}$ Christine Mackerness, ${ }^{1}$ Rebekah Hoskins, ${ }^{1}$ Allison Shiell. ${ }^{1}$ Newcastle upon Tyne Hospitals NHS Trust, Newcastle upon Tyne, UK; ${ }^{2}$ St Oswald's Hospice, Newcastle upon Tyne, UK

\subsection{6/spcare-2021-Hospice.21}

Background Although the North East, North Cumbria region has an active paediatric palliative care network, until recently the region lacked a dedicated palliative care service covering all localities and settings.

Supporting choice in place of death is a core principle of palliative care (National Institute for Health and Care Excellence. End of life care for infants, children and young people, Guidance NG61, 2016, rev. 2019; National Palliative and End of Life Partnership, 2021; Siden, Miller, Straatman, et al., 2008; Wolff, Robert, Sommerer, et al., 2010), however, data for the region demonstrated that very few children with nonmalignant conditions received end of life care at home (Gibson-Smith , Jarvis, Fraser, online first 2020). In 2021 a new regional children's palliative care service was launched, funded by specialist commissioning. We report on the early impact of the service relating to place of death.

Aims and Methods The service aims to improve families' experience by providing choice in place of death through:

- Assisting local paediatric teams with advice and practical help.

- Supporting families with visits and telephone contact.

- Facilitating decision-making and advance care planning.

Robust evaluation has been built into the service from the outset including collection of family and professional feedback. Results In the first six months of the project, the service received over 50 referrals. The palliative care team supported 11 children with end-of-life care and $100 \%$ died in their preferred setting. Six children received end-of-life care at home and the team facilitated five rapid transfers. Feedback from professionals and families highlighted the impact of the new service on enabling end-of-life care in community settings. The service supported children from every part of the region working alongside teams from all the four hospices and many children's clinical teams in the region. Locality debriefs following a child's death consolidated learning and continue to build relationships with local partners.

Conclusion We present evidence that the new children's palliative care service has successfully enabled children to receive consistent end-of-life care across all settings in the region, thus facilitating greater choice for families.

\section{$0-23$ VIEWS AND EXPERIENCES OF YOUNG PEOPLE, PARENTS/CARERS AND PROFESSIONALS OF ADVANCE CARE PLANNING}

Ben Hughes', Katherine Knighting², Mary O'Brien², Anita Flynn². 'University of Bolton, Bolton, UK; ${ }^{2}$ Edge Hill University, Ormskirk, UK

10.1136/spcare-2021-Hospice.22
Background Advance care planning (ACP) for adults has been widely practised in the United States, Canada, Australia, and New Zealand and has been embedded in the United Kingdom (UK) since 2005 (Dixon \& Knapp, 2020; Stein \& Fineberg, 2013; Hughes, O’Brien, Flynn, et al., 2018; Hayhoe, Howe, Gillick, et al., 2011). Approximately 99,000 children and young people in the UK have a life-limiting or life-threatening condition and may benefit from ACP (Fraser, Gibson-Smith, Jarvis, et al., 2020). However, little is understood about engaging young people in their own ACP from the different perspectives of those involved in the process.

Aim(s) To explore the views and experiences of young people, their parents/carers and healthcare professionals in the advance care planning process. Objectives were to explore both optimal timing of ACP discussions and facilitators and barriers to engaging young people in their care planning.

Methods A qualitative multiple case study research design was used to recruit participants, with young people as the unit of analysis. Data were collected using semi-structured interviews and analysed using thematic analysis and critical discourse analysis.

Results Views and experiences were mixed across the case studies. ACP should be initiated when young people are in their mid-teens, their condition is stable, and prior to transition to adult care. Facilitators to engagement were reported to be age- and developmentally-appropriate communication, relationships developed prior to initiating ACP, and support for everyone in the process. Perceived hierarchies of power and potential for disjointed communication and relationships can lead to misperception of ACP, resulting in barriers to engaging young people and negative experiences of the process.

Conclusions Exploration and understanding of views and experiences of different, concurrent participant groups has relevance for policy and practice. Understanding optimal timing of ACP discussions and the pivotal roles of communication, relationships, and organisational structure and culture, can create facilitators or barriers to engaging young people, in their own care planning.

This study was completed as a $\mathrm{PhD}$, which was funded by Edge Hill University. There are no conflicts of interest.

\section{Poster Presentations}

\section{Inequalities of access}

\section{P-1 A POPULATION HEALTH APPROACH TO QUANTIFYING HEALTH INEQUALITIES IN HOSPICE CARE}

Emma Hodges. St Giles Hospice, West Midlands, UK

\subsection{6/spcare-2021-Hospice.23}

Quantifying key elements of hospice care at primary care network level enables more targeted and clear discussions within the emerging Integrated Care Systems. Population health management creates a prime opportunity for hospices, particularly combining specialist care with community development activities. The combination of these factors creates possibly one of the best opportunities for hospices to engage and tackle health inequalities relating to access to palliative care. 